\title{
Criteria for nonclassicality in the prepare-and-measure scenario
}

\author{
Davide Poderini, ${ }^{1}$ Samuraí Brito, ${ }^{2}$ Ranieri Nery $\odot,{ }^{2}$ Fabio Sciarrino, ${ }^{1}$ and Rafael Chaves ${ }^{2,3}$ \\ ${ }^{1}$ Dipartimento di Fisica, Sapienza Università di Roma, Piazzale Aldo Moro 5, I-00185 Roma, Italy \\ ${ }^{2}$ International Institute of Physics, Federal University of Rio Grande do Norte, 59070-405 Natal, Brazil \\ ${ }^{3}$ School of Science and Technology, Federal University of Rio Grande do Norte, 59078-970 Natal, Brazil
}

(Received 1 June 2020; accepted 24 September 2020; published 20 October 2020)

\begin{abstract}
Quantum communication networks involving the preparation, sharing, and measurement of quantum states are ubiquitous in quantum information. Of particular relevance within this context is to understand under which conditions a given quantum resource can give rise to correlations incompatible with a classical explanation. Here we consider the so-called prepare-and-measure scenario, in which a quantum or classical message with bounded dimension is transmitted between two parties. In this scenario we derive criteria witnessing whether a set of quantum states can lead or not to nonclassical correlations. Based on that, we show that quantum resources that can only give rise to classical correlations in the simplest prepare-and-measure scenario can have their nonclassicality witnessed if we increase the number of preparations or measurements.
\end{abstract}

DOI: 10.1103/PhysRevResearch.2.043106

\section{INTRODUCTION}

The incompatibility of quantum predictions with the classical theory of causality $[1,2]$ is at the core of the foundations of quantum theory. A paradigmatic example is Bell's theorem [3], showing that measurements on distant entangled systems cannot be explained by classical models built on the causal assumptions of locality and measurement independence [4,5], generally known as local hidden variable models. From the causal perspective, Bell's theorem refers to a simple causal structure where a source distributes correlations between two distant parties. Generalizations to causal networks of growing size and complexity are attracting much attention lately [6-13]. Despite steady advances both theoretically and experimentally, most recent results about quantum networks refer to Bell-like causal structures, that is, sources distributing correlations between distant noncommunicating parties.

Notwithstanding, communication quantum networks are ubiquitous in quantum information. In a teleportation protocol [14], for instance, shared entanglement plus classical communication allows for the transmission of quantum information. In turn, in a dense-coding protocol [15], entanglement plus quantum communication allows for a more efficient transmission of classical data. A particularly relevant framework in quantum communication is the so-called prepareand-measure (PAM) scenario [16]. In its simplest form there is a preparation device that produces and sends physical systems to a measurement device able to perform different measurements on such systems. The prepare-and-measure

Published by the American Physical Society under the terms of the Creative Commons Attribution 4.0 International license. Further distribution of this work must maintain attribution to the author(s) and the published article's title, journal citation, and DOI. scenario is at the core of random access codes [17-19], informational principles to quantum theory [20,21], in modeling the delayed choice experiment [22-25], and in many different types of semi-device-independent quantum information protocols [31-35]. The PAM scenario is also fundamental in the device-independent estimation of entropy [26] and dimension [16,27-30]. Device independence refers here to the fact that properties of the system can be inferred (for example, its dimension) just from the observational data and without the precise knowledge of the preparation and measurement devices. This is possible because in this scenario one imposes assumptions on the dimension of the prepared system, introducing further constraints for the possible correlations.

Within this context a central question is to decide, given that a certain causal structure is imposed, whether a given quantum resource can give rise to correlations without a classical explanation. In the Bell scenario, a paradigmatic example is the Horodecki criterion [36], testing whether a given two-qubit quantum state can violate the Clauser-HorneShimony-Holt (CHSH) inequality [37]. Extensions of it to more general scenarios [38,39] and other Bell-like causal structures are also known [40,41]. In scenarios involving communication, for instance in quantum teleportation, it is known that all entangled states can lead to nonclassical correlations [42]. This result, however, depends on the tomography of the teleported state, that is, is not device-independent. In fact, to our knowledge, there is no analog of the Horodecki criterion [36] to causal structures involving communication, in particular the prepare-and-measure scenario.

Here, our aim is precisely that: to derive criteria testing whether a given quantum resource can lead to nonclassical correlations in the prepare-and-measure scenario. To that aim we derive a set of necessary and sufficient criteria for the violation of the inequality describing classical correlations in the simplest PAM scenario (with three preparations and two 
(a)

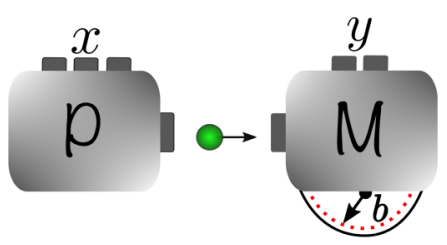

(b)

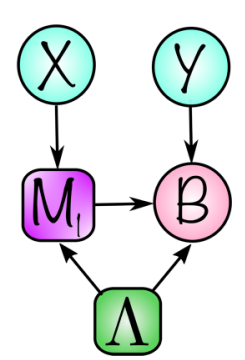

FIG. 1. (a) Black-box representation of the prepare-and-measure scenario. (b) DAG representation of the classical causal structure assuming that the devices might be correlated via $\Lambda$ variable (or via a shared quantum state in a quantum description). The variable $M$ stands for the prepared state and in the quantum case would be represented by a quantum state $\rho_{x}$.

possible measurements) when the dimension of the preparation is bounded [16]. This inequality belongs to a broader class of inequalities bounding the classically attainable correlations in the case of fixed dimension of the prepared system, which were presented in [16] as classical dimension witnesses.

It is the analog in the PAM scenario of what the Horodecki criterion [36] is for the Bell case. Further, we employ these criteria to show a few activation-like results. Namely, resources that can only lead to classical behavior in the simplest PAM scenario can have their nonclassicality revealed by increasing the number of preparations or measurements of the devices. We also analyze the possibility of activation in communication networks having the PAM causal structure as a building block.

The paper is organized as follows. In Sec. II we describe in detail the PAM scenario, its underlying causal structure, and classical as well as quantum representations. In Sec. III we derive the main result of the paper, a set of criteria testing whether a given quantum resource can lead to nonclassicality. It applies to two situations: first, the case where qubit states are prepared but only classical correlations are allowed between the preparation and measurement devices; second, the case where the prepared states are classical but the devices can share quantum correlations. In Sec. IV we present the activation-like results described above. In Sec. V we analyze simple communication networks built from the standard PAM scenario. In Sec. VI we discuss our results and point out future directions. Finally, in the Appendix we provide technical details underlying our results.

\section{THE PREPARE-AND-MEASURE SCENARIO}

The PAM scenario is composed of a preparation and a measurement device (see Fig. 1). The preparation device takes inputs described by a random variable $X$ and depending on these inputs prepares different physical systems. Upon receiving the prepared physical systems, the measurement device also takes inputs described by a random variable $Y$ and depending on these inputs measure different observables and obtain a measurement outcome described by a random variable $B$.

In a classical description, the physical systems being prepared can be described by a random variable $M$, though this variable is not empirically observable, just the measurement results $B=b$ obtained by measurements over it. Generally, the observable data in a PAM experiment are then described by the probability distribution $p(X=x, Y=y, B=$ $b)=p(x, y, b)$. Since $X$ and $Y$ are external to the devices and in control of the experimenter, typically the conditional probability $p(b \mid x y)$ is considered. It is implicit in this description that the input $X$ is not fed directly into the measurement device; that is, all the information the measurement device has about $X$ is mediated by $M$. Furthermore, since the choice of which observable to measure is done after the system has been prepared, $M$ cannot depend on the variable $Y$. Such causal assumption can be graphically represented by a directed acyclic graph (DAG) as shown in Fig. 1(b). Thus, the observable distribution can be decomposed as

$$
p(b \mid x, y)=\sum_{m} p(b \mid m, y) p(m \mid x) .
$$

Notice that unless we bound the dimension of $M$ (the number of possible values it can assume) to be strictly smaller than the cardinality of $X$, the problem becomes trivial since any distribution $p(b \mid x, y)$ can be generated by such causal model. Thus, the dimension of the physical system being prepared is typically bounded (see $[34,35,43]$ for alternative constraints not bounding the dimension of the prepared states).

In a classical description, without loss of generality, any randomness present in the probabilities can be absorbed into latent/hidden variables [1]. Assuming that the preparation and measurement devices are independent (that is, share no correlations), this implies that

$$
p(b \mid x, y)=\sum_{\mu, v, m} p(b \mid m, y, v) p(m \mid x, \mu) p(\mu) p(v) .
$$

Even though in most situations it is reasonable to assume the independence of the devices, this independence leads a nonlinear constraint $p(\mu, v)=p(\mu) p(v)$ implying that the set of distributions $p(b \mid x, y)$ compatible with such model is nonconvex and difficult to be characterized apart from some particular instances [27]. Because of that, in most cases it is assumed that the preparation and measurement devices might be correlated [16], implying that

$$
p(b \mid x, y)=\sum_{\lambda, m} p(b \mid m, y, \lambda) p(m \mid x, \lambda) p(\lambda) .
$$

We now turn our attention to a quantum description of the PAM scenario. Assuming uncorrelated preparation and measurement devices, upon taking input $X=x$ the preparation device prepares quantum systems described by density matrix $\rho_{x}$. In turn, the measurement device is described by a collection of measurement operators $M_{b}^{y}$. Applying Born's rule, the quantum probability in the PAM scenario is then given by

$$
p(b \mid x, y)=\operatorname{Tr}\left(\rho_{x} M_{b}^{y}\right) .
$$

In the case where the devices might be correlated, other quantum descriptions become possible [18]. For instance, the correlations between the devices can be quantum (a shared quantum state) but the states being prepared are classical. In 
this case, the observed probability distribution is given by

$$
p(b \mid x, y)=\sum_{a} \operatorname{Tr}\left[\left(M_{a}^{x} \otimes M_{b}^{y, m(a)}\right) \rho_{A B}\right] .
$$

That is, the preparation and measurement devices share a joint quantum state; the preparation device measures its share obtaining an outcome $a$ and prepares a random variable $m(a)$ that depends on such outcome. Then, the measurement device measures its part of the shared state in a basis that depends not only on its own input $y$ but also on the prepared random variable $m(a)$. Finally, one can also consider the case where both the shared correlations and the prepared states are quantum, as for instance in the dense-coding protocol [15], but we will not consider this case here.

Let us now consider the simplest nontrivial PAM scenario (see Fig. 1), where we have 3 preparations ( $x=1,2,3)$, two possible dichotomic measurements measurements $(y=1,2$ and $b=0,1)$, and where the prepared states are assumed to be two-dimensional ( $m=0,1$, that is, it can be considered a bit). Any correlation compatible with the classical descriptions (2) or (3) respects the inequality [16]

$$
S=E_{11}+E_{12}+E_{21}-E_{22}-E_{31} \leqslant 3,
$$

where $E_{x y}=p(0 \mid x y)-p(1 \mid x y)$ is the expectation value, and $p(b \mid x y)$ represent the probability of having an outcome $b$ with settings $x$ and $y$. Because of the restriction in the dimension of the prepared states, this inequality is refereed as a dimension witness (DW) inequality.

Under the same dimension constraints, quantum correlations violate the $S$ inequality, thus showing the incompatibility of the classical and quantum descriptions. In the quantum case described by (4), considering that the prepared systems are qubits, the maximum quantum violation is given by $S=1+$ $2 \sqrt{2} \approx 3.82$. This is the same maximum violation considering that the prepared states are classical but that the devices share joint entangled states [see (5)].

Next, we will derive a necessary and sufficient criterion for the violation of the inequality (6) by quantum resources and later use this criterion to show the activation-like results by increasing the number of preparations or measurements in the devices. Our criterion can be seen as the analog for the PAM scenario of the Horodecki criterion [36] showing the requirements on the entangled state for the violation of the CHSH inequality in a standard bipartite Bell scenario.

\section{CRITERIA FOR THE QUANTUM VIOLATION OF THE DW INEQUALITY}

Our aim here is to derive a necessary and sufficient criterion for nonclassicality in the simplest possible PAM scenario, that is, with three possible preparations and two measurements. This scenario is fully described by the inequality (6) and its 24 symmetries, corresponding to relabeling of preparation and measurement inputs as well the relabeling of measurement outputs.

As mentioned above, we will consider here two possible quantum realizations of the prepare-and-measure scenario: the first in which the prepared systems are quantum states and the second where such systems are classical random variables but quantum correlations are allowed between the preparation and measurement devices.

\section{A. Preparation device produces quantum states}

Since $\rho_{x}$ is assumed to be 2-dimensional, we can parametrize the quantum states using the Bloch sphere representation for a single qubit,

$$
\rho_{x}=\frac{1}{2}\left(\mathbb{1}+\boldsymbol{r}_{x} \cdot \boldsymbol{\sigma}\right),
$$

where $\sigma=\left(\sigma_{x}, \sigma_{y}, \sigma_{z}\right)$ is the vector with components given by the Pauli matrices.

General two-outcome positive-operator-valued measures (POVMs) are described by operators given by

$$
B_{y}^{b}=\lambda^{b}\left(\mathbb{1}+\boldsymbol{q}_{y}^{b} \cdot \boldsymbol{\sigma}\right),
$$

where $b \in\{0,1\}$, with $\lambda^{0}+\lambda^{1}=1$ and $\lambda^{0} \boldsymbol{q}^{0}+\lambda^{1} \boldsymbol{q}^{1}=0$. However, without loss of generality, since we are interested in the maximum violation of the dimension witness (6) which is linear, we can restrict ourselves to extremal POVMs, which in the case of qubits and two outcomes are simply given by projective measurements

$$
B_{y}^{b}=\frac{1}{2}\left(\mathbb{1} \pm \boldsymbol{q}_{y} \cdot \boldsymbol{\sigma}\right),
$$

with $\left\|\boldsymbol{q}_{y}\right\|=1$. In terms of the Bloch vectors $\boldsymbol{r}_{x}$ and $\boldsymbol{q}_{y}$, the probability (4) becomes

$$
\begin{aligned}
p(b \mid x, y) & =\operatorname{Tr}\left(\rho_{x} B_{y}^{b}\right) \\
& =\frac{1}{4} \operatorname{Tr}\left[\mathbb{1}\left(1+\boldsymbol{r}_{x} \cdot \boldsymbol{q}_{y}\right)+\boldsymbol{r}_{x} \cdot \boldsymbol{\sigma}+\boldsymbol{q}_{y} \cdot \boldsymbol{\sigma} \pm i \boldsymbol{r}_{x} \times \boldsymbol{q}_{y}\right] \\
& =\frac{1}{2}\left(\mathbb{1} \pm \boldsymbol{r}_{x} \cdot \boldsymbol{q}_{y}\right) .
\end{aligned}
$$

Substituting this expression in the correlators $E_{x y}$ we obtain

$$
E_{x y}=\operatorname{Tr}\left(\rho_{x} B_{y}^{0}\right)-\operatorname{Tr}\left(\rho_{x} B_{y}^{1}\right)=\boldsymbol{r}_{x} \cdot \boldsymbol{q}_{y} .
$$

Then, inequality (6) becomes

$$
S=\boldsymbol{q}_{1} \cdot\left(\boldsymbol{r}_{1}+\boldsymbol{r}_{2}-\boldsymbol{r}_{3}\right)+\boldsymbol{q}_{2} \cdot\left(\boldsymbol{r}_{1}-\boldsymbol{r}_{2}\right) \leqslant 3 .
$$

Clearly, the maximum for this expression is obtained if we choose $\boldsymbol{q}_{1}$ and $\boldsymbol{q}_{2}$ as representing projective measurements with the corresponding vectors on the Bloch sphere parallel to the directions of $\boldsymbol{r}_{1}+\boldsymbol{r}_{2}-\boldsymbol{r}_{3}$ and $\boldsymbol{r}_{1}-\boldsymbol{r}_{2}$, respectively.

Notice that so far we have considered only one of the 24 symmetries of inequality (6). Relabeling of the measurement inputs or outputs does not change the reasoning presented above, so only the relabeling of the preparation inputs has to be considered. So a given set of states $\left\{\rho_{1}, \rho_{2}, \rho_{3}\right\}$ cannot violate any of the symmetries of inequality (6) if and only if all the following constraints are fulfilled:

$$
\begin{gathered}
\left\|\boldsymbol{r}_{1}+\boldsymbol{r}_{2}-\boldsymbol{r}_{3}\right\|+\left\|\boldsymbol{r}_{1}-\boldsymbol{r}_{2}\right\| \leqslant 3, \\
\left\|\boldsymbol{r}_{1}+\boldsymbol{r}_{3}-\boldsymbol{r}_{2}\right\|+\left\|\boldsymbol{r}_{1}-\boldsymbol{r}_{3}\right\| \leqslant 3, \\
\left\|\boldsymbol{r}_{3}+\boldsymbol{r}_{2}-\boldsymbol{r}_{1}\right\|+\left\|\boldsymbol{r}_{3}-\boldsymbol{r}_{2}\right\| \leqslant 3,
\end{gathered}
$$

where we have considered all permutations of $\boldsymbol{r}_{1}, \boldsymbol{r}_{2}, \boldsymbol{r}_{3}$

If we limit ourselves to pure states, then we can express the sum and difference of $\boldsymbol{r}_{1}, \boldsymbol{r}_{2}$, and $\boldsymbol{r}_{3}$ as

$$
\begin{array}{ll}
\boldsymbol{r}_{1}+\boldsymbol{r}_{2}=2 \boldsymbol{u}_{1} \cos \theta_{1}, & \boldsymbol{r}_{1}-\boldsymbol{r}_{2}=2 \boldsymbol{u}_{2} \sin \theta_{1}, \\
\boldsymbol{r}_{2}+\boldsymbol{r}_{3}=2 \boldsymbol{v}_{1} \cos \theta_{2}, & \boldsymbol{r}_{2}-\boldsymbol{r}_{3}=2 \boldsymbol{v}_{2} \sin \theta_{2},
\end{array}
$$




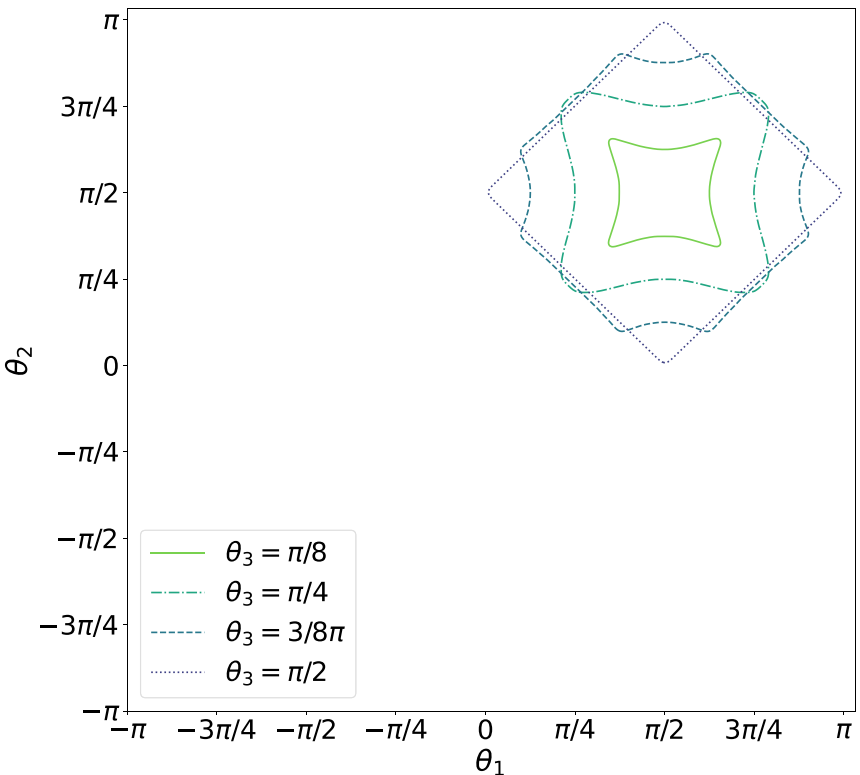

FIG. 2. Plot showing the condition (15). The plot shows the values of $\theta_{1}$ and $\theta_{2}$ for which the corresponding states cannot violate the inequality (6). In particular, states belonging to the area outside the dotted, dashed, dash-dotted, and solid lines cannot violate (6) for $\theta_{3}$ equal to $\pi / 2,3 \pi / 8, \pi / 4$, and $\pi / 8$, respectively.

$$
\boldsymbol{r}_{1}+\boldsymbol{r}_{3}=2 \boldsymbol{w}_{1} \cos \theta_{3}, \quad \boldsymbol{r}_{1}-\boldsymbol{r}_{3}=2 \boldsymbol{w}_{2} \sin \theta_{3},
$$

where $\left(\boldsymbol{u}_{1}, \boldsymbol{u}_{2}\right),\left(\boldsymbol{v}_{1}, \boldsymbol{v}_{2}\right)$, and $\left(\boldsymbol{w}_{1}, \boldsymbol{w}_{2}\right)$ are couples of orthonormal vectors. Notice that the angles $2 \theta_{1}, 2 \theta_{2}$, and $2 \theta_{3}$ represent the angles between the couple vectors $\left(\boldsymbol{r}_{1}, \boldsymbol{r}_{2}\right),\left(\boldsymbol{r}_{2}, \boldsymbol{r}_{3}\right)$, and $\left(\boldsymbol{r}_{1}, \boldsymbol{r}_{3}\right)$, respectively [this can be easily shown by squaring the first equation for each row in (14) obtaining $\boldsymbol{r}_{1} \cdot \boldsymbol{r}_{2}=\cos 2 \theta_{1}, \boldsymbol{r}_{2} \cdot \boldsymbol{r}_{3}=\cos 2 \theta_{2}$, and $\boldsymbol{r}_{1} \cdot \boldsymbol{r}_{3}=$ $\cos 2 \theta_{3}$ ]. Squaring inequalities (13) and using equations (14), the above conditions can be rewritten as

$$
\begin{aligned}
& 2 \cos ^{2} \theta_{1}-\left(\cos 2 \theta_{2}+\cos 2 \theta_{3}\right) / 2+3 \sin \theta_{1} \leqslant 3, \\
& 2 \cos ^{2} \theta_{2}-\left(\cos 2 \theta_{1}+\cos 2 \theta_{3}\right) / 2+3 \sin \theta_{2} \leqslant 3, \\
& 2 \cos ^{2} \theta_{3}-\left(\cos 2 \theta_{1}+\cos 2 \theta_{2}\right) / 2+3 \sin \theta_{3} \leqslant 3 .
\end{aligned}
$$

To illustrate, the region of violating states for different values of $\theta_{1}, \theta_{2}$, and $\theta_{3}$ is shown in Fig. 2.

\section{B. Prepared states are classical and shared correlations are quantum}

In this case, the preparation and measurement devices share a quantum state $\rho_{A B}$ giving rise to correlations according to (5). As in the Horodecki criterion [36], we limit ourselves to 2-qubit bipartite quantum states that can be can parametrized as

$$
\rho_{A B}=\frac{1}{4} \sum_{i, j=0}^{3} w_{i j} \sigma_{i} \otimes \sigma_{j},
$$

where $\sigma_{0}=\mathbb{1}$ is the identity operator and $\sigma_{i}$ for $i=1,2,3$ the Pauli matrices. In order to represent a density matrix, $w_{i j}$ has to satisfy some additional properties. Imposing the unitary trace condition gives us $w_{00}=1$ and from Hermiticity we have that the elements $w_{i j}$ have to be real. The condition for satisfying positivity is more involved, and it is described in [44].

We consider measurement operators $A_{x}$ and $B_{y m}$, for the preparation and measurement devices, respectively, given by

$$
\begin{aligned}
A_{x}^{a} & =\frac{1}{2}\left(\mathbb{1} \pm \boldsymbol{q}_{x}^{A} \cdot \boldsymbol{\sigma}\right), \\
B_{y m}^{b} & =\frac{1}{2}\left(\mathbb{1} \pm \boldsymbol{q}_{y m}^{B} \cdot \boldsymbol{\sigma}\right) .
\end{aligned}
$$

Defining the label $m$ as $m=(-1)^{a}$, the expectation values $E_{x y}$ appearing in the inequality (6) can be written as

$$
E_{x y}=\frac{1}{2} \sum_{m=-1}^{1} \sum_{j=1}^{3}\left[w_{0 j}+m \sum_{i=1}^{3} w_{i j}\left(q_{x}^{A}\right)^{i}\right]\left(q_{y m}^{B}\right)^{j} .
$$

Substituting this in (6) we obtain

$$
\begin{aligned}
S= & \frac{1}{2}\left[\left(\boldsymbol{q}_{1}^{A}+\boldsymbol{q}_{2}^{A}-\boldsymbol{q}_{3}^{A}\right) T\left(\boldsymbol{q}_{1+}^{B}-\boldsymbol{q}_{1-}^{B}\right)\right. \\
& \left.+\left(\boldsymbol{q}_{1}^{A}-\boldsymbol{q}_{2}^{A}\right) T\left(\boldsymbol{q}_{2+}^{B}-\boldsymbol{q}_{2-}^{B}\right)+\left(\boldsymbol{q}_{1+}^{B}+\boldsymbol{q}_{1-}^{B}\right) \cdot \boldsymbol{s}\right],
\end{aligned}
$$

where $T$ represents the $3 \times 3$ matrix with elements $w_{i j}$ for $i, j \in\{1,2,3\}$, and the vector $s$ is defined as $s=$ $\left(w_{01}, w_{02}, w_{03}\right)$.

\section{The $s=0$ case}

To simplify the problem we first focus on the case where $\boldsymbol{s}=(0,0,0)$. This corresponds to the case where our 2-qubit state appears as completely mixed if we trace out one the parties, as for instance, in a maximally entangled state. In this case the last term in $S$ [as described by (20)] is zero, and it is clear that the maximum in $\boldsymbol{q}_{y m}^{B}$ is reached when the combinations $\boldsymbol{q}_{y+}^{B}-\boldsymbol{q}_{y-}^{B}$ are parallel to $\left(\boldsymbol{q}_{1}^{A}+\boldsymbol{q}_{2}^{A}-\boldsymbol{q}_{3}^{A}\right) T$ and $\left(\boldsymbol{q}_{1}^{A}-\boldsymbol{q}_{2}^{A}\right) T$ for $y=1$ and $y=2$, respectively. Moreover we can maximize on $\boldsymbol{q}_{3}^{A}$ choosing it to be parallel to $\boldsymbol{q}_{1}^{A}+\boldsymbol{q}_{2}^{A}$. Inequality (6) can then be expressed as

$$
S=\alpha\left\|T\left(\boldsymbol{q}_{1}^{A}+\boldsymbol{q}_{2}^{A}\right)\right\|+\left\|T\left(\boldsymbol{q}_{1}^{A}-\boldsymbol{q}_{2}^{A}\right)\right\| \leqslant 3,
$$

where $\alpha=1+1 /\left\|\boldsymbol{q}_{1}^{A}+\boldsymbol{q}_{2}^{A}\right\|$. Again, without loss of generality, we can restrict ourselves to the case of projective measurements, that is, $\left\|\boldsymbol{q}_{1}^{A}\right\|=\left\|\boldsymbol{q}_{2}^{A}\right\|=1$. As in the previous section we express the sum and difference of the vectors $\boldsymbol{q}_{1}^{A}$ and $\boldsymbol{q}_{2}^{A}$ as $\left(\boldsymbol{q}_{1}^{A}+\boldsymbol{q}_{2}^{A}\right)=2 \boldsymbol{u}_{1} \cos \theta$ and $\left(\boldsymbol{q}_{1}^{A}-\boldsymbol{q}_{2}^{A}\right)=2 \boldsymbol{u}_{2} \sin \theta$, where $\boldsymbol{u}_{1}, \boldsymbol{u}_{2}$ are two arbitrary orthonormal vectors. In particular, to maximize $S$, we choose them to be in the direction of the eigenvectors, corresponding to the two largest eigenvalues $\lambda_{1}, \lambda_{2}$ of the symmetric matrix $U=T^{\top} T$, with $\lambda_{1} \geqslant \lambda_{2}$. Maximizing the resulting expression on $\theta$ gives rise to (see Appendix for an alternative derivation)

$$
S=2 \sqrt{\lambda_{1}+\lambda_{2}}+\sqrt{\lambda_{1}} \leqslant 3 .
$$

If the matrix $T_{i j}$ describing the 2-qubit state satisfies this inequality, then such state cannot violate the dimensional witness (6).

It is worth noting that this criterion is similar to the Horodecki criterion [36]

$$
M\left(\rho_{\lambda}\right)=\lambda_{1}+\lambda_{2} \leqslant 1,
$$

for the CHSH inequality [37], however, less restrictive. Indeed, if we plot the region of violation of the $\mathrm{CHSH}$ and the 


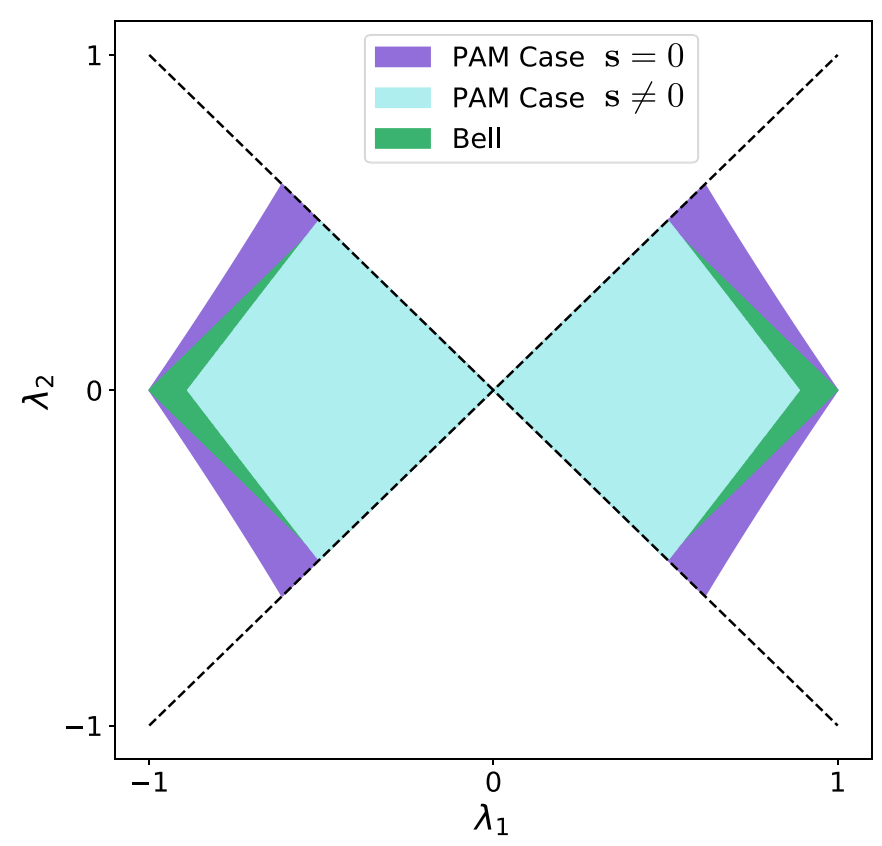

FIG. 3. Plot of the region described by the criteria (22) and (31) in the space of the eigenvalues $\lambda_{1}, \lambda_{2}$ of $U=T^{\dagger} T$ where $T$ is the matrix describing a 2 -qubit state. The green area represents the region fulfilling the Horodecki criterion [see Eq. (23)] for the Bell scenario [36].

PAM inequalities as a function of $\lambda_{1}$ and $\lambda_{2}$, we see that the former is always included in the latter (see Fig. 3).

\section{The $s \neq 0$ case}

We start rewriting (20) as

$$
S=\frac{1}{2}\left[(\boldsymbol{c} T+\boldsymbol{s}) \boldsymbol{q}_{1+}^{B}-(\boldsymbol{c} T-\boldsymbol{s}) \boldsymbol{q}_{1-}^{B}+\boldsymbol{d} T\left(\boldsymbol{q}_{2+}^{B}-\boldsymbol{q}_{2-}^{B}\right)\right],
$$

where $\boldsymbol{c}=\boldsymbol{q}_{1}^{A}+\boldsymbol{q}_{2}^{A}-\boldsymbol{q}_{3}^{A}$ and $\boldsymbol{d}=\boldsymbol{q}_{1}^{A}-\boldsymbol{q}_{2}^{A}$. As before, we maximize on the vectors $\boldsymbol{q}_{1 \pm}^{B}, \boldsymbol{q}_{2 \pm}^{B}$ obtaining

$$
S=\frac{1}{2}(\|c T+s\|+\|c T-s\|)+\|d T\| .
$$

Instead of maximizing on $\boldsymbol{c}$ and $\boldsymbol{d}$ directly, we can find an upper and lower bound for this expression by maximizing or minimizing on the vector $s$. Equivalently, we maximize or minimize the quantity

$$
\begin{aligned}
\| \boldsymbol{r} & +\boldsymbol{s}\|+\| \boldsymbol{r}-\boldsymbol{s} \| \\
& =\sqrt{r^{2}+s^{2}+2 r s \cos \theta}+\sqrt{r^{2}+s^{2}-2 r s \cos \theta},
\end{aligned}
$$

for two vectors in $\mathbb{R}^{3}$ (with $\boldsymbol{r}=\boldsymbol{c} T$ ). We have $\theta=0$ for the minimum and $\theta=\pi / 2$ for the maximum, corresponding to the values $2 r$ and $2 \sqrt{r^{2}+s^{2}}$, respectively. Hence the minimum corresponds to the case $s=0$, already solved in the previous section, while for the maximum, we get

$$
S=2 \sin \theta\left\|\boldsymbol{u}_{2} T\right\|+\sqrt{(2 \cos \theta+1)^{2}\left\|\boldsymbol{u}_{1} T\right\|^{2}+s^{2}},
$$

where $\left(\boldsymbol{r}_{1}+\boldsymbol{r}_{2}\right)=2 \boldsymbol{u}_{1} \cos \theta$ and $\left(\boldsymbol{r}_{1}-\boldsymbol{r}_{2}\right)=2 \boldsymbol{u}_{2} \cos \theta$, with $\boldsymbol{u}_{1}, \boldsymbol{u}_{2}$ being two arbitrary orthonormal vectors. Calling $\lambda_{1}, \lambda_{2}$ the absolute value of the two largest eigenvalues of the symmetric matrix $U=T^{\top} T$, with $\lambda_{1} \geqslant \lambda_{2}$, and maximizing also on the norm of $s$, we obtain

$$
S \leqslant 2 \sin \theta \sqrt{\lambda_{2}}+\sqrt{(2 \cos \theta+1)^{2} \lambda_{1}+1} .
$$

Deriving this expression in $\theta$ we obtain the condition

$$
\tan ^{2} \theta=\frac{\lambda_{2}}{\lambda_{1}} \frac{(2 \cos \theta+1)^{2} \lambda_{1}+1}{(2 \cos \theta+1)^{2} \lambda_{1}},
$$

which leads to the following quartic equation in $x=\cos \theta$,

$$
x^{4}+x^{3}-x^{2}(b-1 / 4)-a x-a / 4=0,
$$

where $a=\lambda_{1} /\left(\lambda_{1}+\lambda_{2}\right)$ and $b=\lambda_{2} / 4 \lambda_{1}\left(\lambda_{1}+\lambda_{2}\right)-a$. This can be solved (either numerically or analytically) for given values of $\lambda_{1}, \lambda_{2}$. Calling $\bar{x}$ the solution that maximizes $S$, we obtain the condition

$$
S \leqslant S_{\max }\left(\lambda_{1}, \lambda_{2}\right)=2 \sqrt{\left(1-\bar{x}^{2}\right) \lambda_{2}}+\sqrt{(2 \bar{x}+1)^{2} \lambda_{1}+1} .
$$

Using this condition we can exclude that a state can violate the inequality for the PAM scenario for any $s$ if we have $S_{\max }\left(\lambda_{1}, \lambda_{2}\right) \leqslant 3$. In Fig. 3 the region of violation for this criterion is illustrated and compared to the Horodecki criterion [36].

\section{NONCLASSICALITY ACTIVATION IN THE PREPARE-AND-MEASURE SCENARIO}

We will consider here two kinds of activation results in the PAM scenario: first, considering that the preparation and measurement devices share no quantum correlations but that the prepared states are quantum (see Sec. III A); second, considering shared quantum states between the preparation and measurement devices but assuming that the prepared states are classical (see Sec. III B). Considering the first scenario, we have derived a necessary and sufficient condition for a set of three prepared states to be able to display nonclassicality in the simplest scenario where two possible measurements are performed. Similarly, in the second scenario we also have a necessary and sufficient criterion showing whether a given shared quantum state can lead to nonclassical correlations in the simplest PAM scenario.

Using these criteria we will show that states that can only display classical correlations in the simplest scenario can have their nonclassicality activated when (a) we increase the number of possible measurements or (b) we increase the number of prepared states.

\section{A. Activation by increasing the number of measurements}

We will focus here on the PAM scenario with three preparations and three measurements [see Fig. 4(a)]. To that aim, as explained in the Appendix, we have obtained the full description of this scenario in terms of linear inequalities. To our aim, we consider the inequality valid for the classical case (considering that the message is a bit and classical correlations between the devices) given by

$$
S_{1}=E_{11}+E_{12}-E_{22}+E_{23}-E_{31}-E_{33} \leqslant 4 .
$$

The activation results we observe in this scenario and described below are conceptually similar to what has been obtained in the context of a Bell scenario. In [45] it has 
(a)

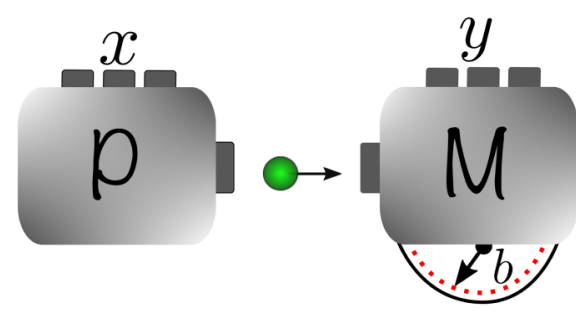

(b)

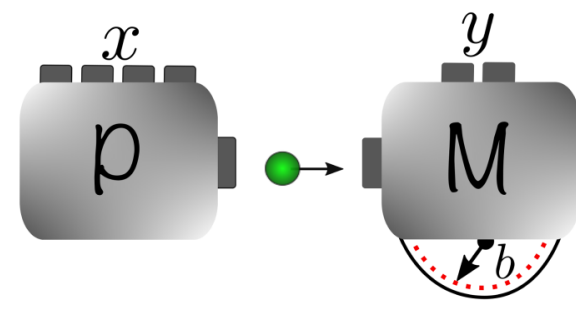

FIG. 4. Black-box representation of the prepare-and-measure scenario with (a) three preparations and three measurements and (b) four preparations and two measurements. See Fig. 1(b) for the corresponding causal structure.

been shown that quantum states that are local in the simplest Bell scenario (two parties with two inputs and outputs), and therefore do not violate the Horodecki criterion [36] for the CHSH inequality [37], can violate a new inequality when one extra measurement input is considered.

\section{Activating the nonclassicality of a set of qubit states}

The goal is to consider a set of three prepared qubit states that cannot violate the necessary and sufficient criteria Eq. (13) when only two measurements are performed but do so when three measurements are performed. By brute-force optimization we have searched for a set of three states that violate inequality (32) while not violating the criteria Eq. (13). The measurement bases are given by

$$
\left|\psi_{y}\right\rangle=\cos \theta_{y}|0\rangle+e^{i \phi_{y}} \sin \left(\theta_{y}\right)|1\rangle,
$$

such that $M_{+}^{y}=\left|\psi_{y}\right\rangle\left\langle\psi_{y}\right|$ and $M_{-}^{y}=\mathbb{1}-\left|\psi_{y}\right\rangle\left\langle\psi_{y}\right|$. The quantum states are parametrized as $\rho_{x}=\frac{1}{2}\left(\mathbb{1}+\boldsymbol{r}_{x} \cdot \boldsymbol{\sigma}\right)$. We optimized over all $\theta_{y}, \phi_{y}$, and $\boldsymbol{r}_{x}$ and we found a set of parameters that satisfies the criterion Eq. (13) and violates the inequality Eq. (32) with $S_{1}=4.17691$. The states and measurement basis achieving that are given by

$$
\begin{aligned}
\left|\psi_{1}\right\rangle=\cos \frac{\pi}{6}|0\rangle+\sin \frac{\pi}{6}|1\rangle, \\
\left|\psi_{2}\right\rangle=|0\rangle, \\
\left|\psi_{3}\right\rangle=\cos \frac{\pi}{3}|0\rangle+\sin \frac{\pi}{3}|1\rangle, \\
\rho_{1}=\frac{1}{2}\left[\mathbb{1}+0.40194 \sigma_{x}+0.696151 \sigma_{z}\right], \\
\rho_{2}=\frac{1}{2}\left[\mathbb{1}+0.40194 \sigma_{x}-0.696151 \sigma_{z}\right], \\
\rho_{3}=\frac{1}{2}\left[\mathbb{1}+0.80388 \sigma_{x}\right] .
\end{aligned}
$$

That is, $\theta_{1}=\pi / 6, \theta_{2}=0, \theta_{3}=\pi / 3, \phi_{1}=\phi_{2}=\phi_{3}=0, \boldsymbol{r}_{1}=$ $a \hat{x}+b \hat{z}, \boldsymbol{r}_{2}=a \hat{x}-b \hat{z}, \boldsymbol{r}_{3}=2 a \hat{x}$, with $a=0.401824$ and $b=$ 0.696151 . Notice that the prepared states are mixed. When restricting to preparations that are pure quantum states, we could not find the same sort of activation results.

\section{Activating the nonclassicality of an entangled quantum state}

A similar result holds if we consider the cases of classical message and a quantum shared state between the preparation and measurement (called Alice and Bob) devices. In this case, we want to find a violation of (32) while enforcing that the criterion (31) is respected. Bob's measurement will depend on Alice's output (a classical message). The shared state $\rho_{A B}$ has the general form given by $\rho_{A B}=\frac{1}{4} \sum_{i j=0}^{3} w_{i j} \sigma_{i} \otimes \sigma_{j}$ with measurements given by $A_{x}^{a}=\frac{1}{2}\left[\mathbb{1}+(-1)^{a} \boldsymbol{q}_{x}^{A} \cdot \boldsymbol{\sigma}\right]$ and by $B_{x, a}^{b}=\frac{1}{2}\left[\mathbb{1}+(-1)^{b} \boldsymbol{q}_{x, a}^{B} \cdot \boldsymbol{\sigma}\right]$. By numerical optimization we could violate inequality (32) up to $S_{1}=4.1755$, using the state

$$
\begin{aligned}
\rho_{A B}= & \frac{1}{2}\left[\mathbb{1} \otimes \mathbb{1}-w_{11} \sigma_{x} \otimes \sigma_{x}-w_{22} \sigma_{y} \otimes \sigma_{y}\right. \\
& \left.+w_{33} \sigma_{z} \otimes \sigma_{z}\right],
\end{aligned}
$$

where $w_{11}=0.801085, \quad w_{22}=-0.749342, \quad$ and $w_{33}=$ 0.829312 , and measurements given by

$$
\begin{aligned}
& A_{0}^{a}=\frac{1}{2}\left[\mathbb{1}-(-1)^{a}\left(\delta_{1} \sigma_{x}+\delta_{2} \sigma_{z}\right)\right], \\
& A_{1}^{a}=\frac{1}{2}\left[\mathbb{1}+(-1)^{a}\left(\delta_{3} \sigma_{x}-\delta_{4} \sigma_{z}\right)\right], \\
& A_{2}^{a}=\frac{1}{2}\left[\mathbb{1}+(-1)^{a}\left(\delta_{5} \sigma_{x}+\delta_{6} \sigma_{z}\right)\right], \\
& B_{0,0}^{b}=\frac{1}{2}\left[\mathbb{1}-(-1)^{b}\left(\gamma_{1} \sigma_{x}+\gamma_{2} \sigma_{z}\right)\right], \\
& B_{0,1}^{b}=\frac{1}{2}\left[\mathbb{1}+(-1)^{b}\left(\gamma_{1} \sigma_{x}-\gamma_{2} \sigma_{z}\right)\right], \\
& B_{1,0}^{b}=\frac{1}{2}\left[\mathbb{1}-(-1)^{b}\left(\gamma_{3} \sigma_{x}-\gamma_{4} \sigma_{z}\right)\right], \\
& B_{1,1}^{b}=\frac{1}{2}\left[\mathbb{1}+(-1)^{b}\left(\gamma_{3} \sigma_{x}-\gamma_{4} \sigma_{z}\right)\right], \\
& B_{2,0}^{b}=\frac{1}{2}\left[\mathbb{1}+(-1)^{b}\left(\gamma_{5} \sigma_{x}-\gamma_{6} \sigma_{z}\right)\right], \\
& B_{2,1}^{b}=\frac{1}{2}\left[\mathbb{1}-(-1)^{b}\left(\gamma_{5} \sigma_{x}-\gamma_{6} \sigma_{z}\right)\right],
\end{aligned}
$$

where $\quad \delta_{1}=0.896959, \quad \delta_{2}=0.433825, \quad \delta_{3}=0.837424$, $\delta_{4}=0.533438, \quad \delta_{5}=0.0595357, \quad \delta_{6}=0.967263, \quad \gamma_{1}=$ $0.550518, \gamma_{2}=0.834823, \gamma_{3}=0.998237, \gamma_{4}=0.0593534$, $\gamma_{5}=0.447719$, and $\gamma_{6}=0.894174$.

\section{B. Activation by increasing the number of preparations}

Here we consider a different kind of activation. We keep the number of measurements fixed but increase the number of preparations to four [see Fig. 4(b)]. To that aim, as described in detail in the Appendix, we have fully characterized the inequalities for this scenario. To find activation it is sufficient to consider the inequality

$$
\begin{aligned}
S_{2}= & E_{11}-E_{12}-E_{21}+E_{22} \\
& -E_{31}-E_{32}+E_{41}+E_{42} \leqslant 4 .
\end{aligned}
$$

\section{Activating the nonclassicality of a set of qubit states}

Activation in this scenario means that any three of the four states are classical [that is, do not violate the criterion 
Eq. (13)] but can have the nonclassicality activated if all four states are considered altogether.

As before we consider measurements parametrized by (33) and generic prepared qubit state of the form $\rho_{x}=\frac{1}{2}\left(\mathbb{1}+\boldsymbol{r}_{x}\right.$. $\boldsymbol{\sigma})$. By performing a brute-force optimization over $\theta_{y}, \phi_{y}$, and $r_{x}$ we found a set of four states such that any three of them satisfy the criteria (13) but violate inequality (46) up to $S_{2}=$ 4.64756. The states and measurement basis are given by

$$
\begin{aligned}
\left|\psi_{1}\right\rangle & =|1\rangle, \\
\left|\psi_{2}\right\rangle & =\cos \frac{\pi}{4}|0\rangle+\sin \frac{\pi}{4}|1\rangle, \\
\rho_{1} & =\frac{1}{2}\left[\mathbb{1}-0.580945\left(\sigma_{x}+\sigma_{z}\right)\right], \\
\rho_{2} & =\frac{1}{2}\left[\mathbb{1}+0.580945\left(\sigma_{x}+\sigma_{z}\right)\right], \\
\rho_{3} & =\frac{1}{2}\left[\mathbb{1}-0.580945\left(\sigma_{x}-\sigma_{z}\right)\right], \\
\rho_{4} & =\frac{1}{2}\left[\mathbb{1}+0.580945\left(\sigma_{x}-\sigma_{z}\right)\right] .
\end{aligned}
$$

These correspond to $\theta_{1}=\pi / 2, \theta_{2}=\pi / 4, \phi_{1}=0, \phi_{2}=2 \pi$, $\boldsymbol{r}_{1}=-a(\hat{x}+\hat{z}), \boldsymbol{r}_{2}=a(\hat{x}+\hat{z}), \boldsymbol{r}_{3}=a(-\hat{x}+\hat{z}), \boldsymbol{r}_{4}=a(\hat{x}-$ $\hat{z})$, with $a=0.580945$. Notice that the prepared states are not pure. Imposing that the prepared states are pure we obtain a smaller violation of $S=4.49$, with measurement and states given by

$$
\begin{aligned}
\left|\psi_{1}\right\rangle & =|1\rangle, \\
\left|\psi_{2}\right\rangle & =\cos \frac{\pi}{4}|0\rangle+\sin \frac{\pi}{4}|1\rangle, \\
\rho_{1} & =\frac{1}{2}\left[\mathbb{1}-0.561325\left(\sigma_{x}+\sigma_{z}\right)-0.608135 \sigma_{y}\right], \\
\rho_{2} & =\frac{1}{2}\left[\mathbb{1}+0.561325\left(\sigma_{x}+\sigma_{z}\right)-0.608135 \sigma_{y}\right], \\
\rho_{3} & =\frac{1}{2}\left[\mathbb{1}-0.561325\left(\sigma_{x}-\sigma_{z}\right)-0.608135 \sigma_{y}\right], \\
\rho_{4} & =\frac{1}{2}\left[\mathbb{1}+0.561325\left(\sigma_{x}-\sigma_{z}\right)-0.608135 \sigma_{y}\right] .
\end{aligned}
$$

Differently from the scenario with an increased number of measurements discussed before, there is no analog of this kind of activation in a Bell scenario, since in the Bell case there is a single preparation. The closest analog we can identify in this case regards the concept of joint measurability and the fact that it is not transitive, that is, subsets of a given set of operators that are jointly measurable, but this does not imply full joint measurability (see [46], for instance). In our case, any subset of three prepared states is classical but the four altogether are not.

\section{Activating the nonclassicality of an entangled quantum state}

We also could find activation in the scenario given by Fig. 1(b) with a classical message and a quantum shared state between preparation and measurement (called Alice and Bob) devices. Here, we want to find a violation of (46) while enforcing that the criterion (31) is respected. By numerical optimization we found a violation of inequality (46) up to (a)

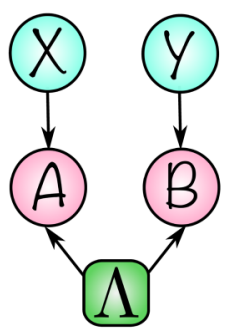

(b)

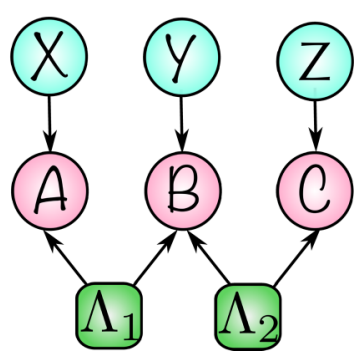

FIG. 5. (a) Causal structure for the paradigmatic bipartite Bell scenario. It can be understood as a preparation device that always prepares the same state that is then sent to two measurement devices (one for Alice, another for Bob). (b) The causal structure underlying the entanglement swapping experiment. Notice that if either Alice or Charlie is traced out, we recover the usual bipartite Bell scenario.

$S_{2}=4.64756$. In our optimization the shared state $\rho_{A B}$ between Alice and Bob has the general form given by $\rho_{A B}=$ $\frac{1}{4} \sum_{i j=0}^{3} w_{i j} \sigma_{i} \otimes \sigma_{j}$ and is given by

$$
\rho_{A B}=\frac{1}{2}\left[\mathbb{1} \otimes \mathbb{1}-w_{1}\left(\sigma_{x} \otimes \sigma_{x}+\sigma_{z} \otimes \sigma_{z}\right)-w_{2} \sigma_{y} \otimes \sigma_{y}\right],
$$

with $w_{11}=w_{33}=0.929299$ and $w_{22}=0.866692$. The measurements are written as $A_{x}^{a}=\frac{1}{2}\left[\mathbb{1}+(-1)^{a} \boldsymbol{q}_{x}^{A} \cdot \boldsymbol{\sigma}\right]$ and $B_{x, a}^{b}=$ $\frac{1}{2}\left[\mathbb{1}+(-1)^{b} \boldsymbol{q}_{x, a}^{B} \cdot \boldsymbol{\sigma}\right]$ and are given by

$$
\begin{aligned}
& A_{0}^{a}=\frac{1}{2}\left[\mathbb{1}+(-1)^{a}\left(\delta_{1} \sigma_{x}+\delta_{2} \sigma_{z}\right)\right], \\
& A_{1}^{a}=\frac{1}{2}\left[\mathbb{1}-(-1)^{a}\left(\delta_{1} \sigma_{x}+\delta_{2} \sigma_{z}\right)\right], \\
& A_{2}^{a}=\frac{1}{2}\left[\mathbb{1}+(-1)^{a}\left(\delta_{2} \sigma_{x}-\delta_{1} \sigma_{z}\right)\right], \\
& A_{3}^{a}=\frac{1}{2}\left[\mathbb{1}-(-1)^{a}\left(\delta_{2} \sigma_{x}-\delta_{1} \sigma_{z}\right)\right], \\
& B_{0,0}^{b}=\frac{1}{2}\left[\mathbb{1}-(-1)^{b}\left(\gamma_{1} \sigma_{x}+\gamma_{2} \sigma_{z}\right)\right], \\
& B_{0,1}^{b}=\frac{1}{2}\left[\mathbb{1}+(-1)^{b}\left(\gamma_{1} \sigma_{x}+\gamma_{2} \sigma_{z}\right)\right], \\
& B_{1,0}^{b}=\frac{1}{2}\left[\mathbb{1}+(-1)^{b}\left(\gamma_{2} \sigma_{x}-\gamma_{1} \sigma_{z}\right)\right], \\
& B_{1,1}^{b}=\frac{1}{2}\left[\mathbb{1}-(-1)^{b}\left(\gamma_{2} \sigma_{x}-\gamma_{1} \sigma_{z}\right)\right],
\end{aligned}
$$

where $\delta_{1}=0.881693, \delta_{2}=0.0650085, \gamma_{1}=0.653198$, and $\gamma_{2}=0.757187$.

\section{BUILDING BLOCKS FOR NETWORKS OF GROWING SIZE AND COMPLEXITY}

In the context of Bell nonlocality it is known that entangled states that are local in the standard Bell causal structure [see Fig. 5(a)] can have their nonlocality activated when distributed into a network. For instance, a state that does not violate the $\mathrm{CHSH}$ inequality can do so when copies of it are distributed in an entanglement swapping network [47-49] [see Fig. 5(b)]. Considering an increasing number of copies and networks of growing size, even stronger activation results become possible. For instance, in [50] it has been shown that a local entangled state [that is, not violating any Bell inequality in the bipartite network of Fig. 5(a)] becomes nonlocal when 
(a)

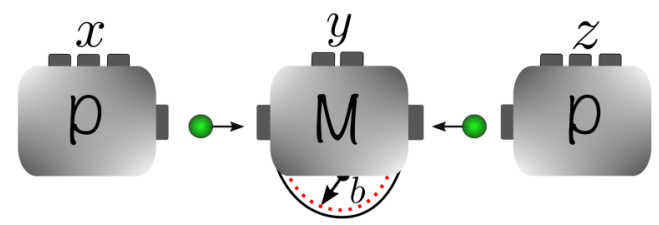

(b)

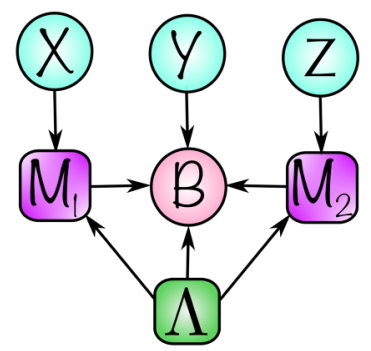

(c)

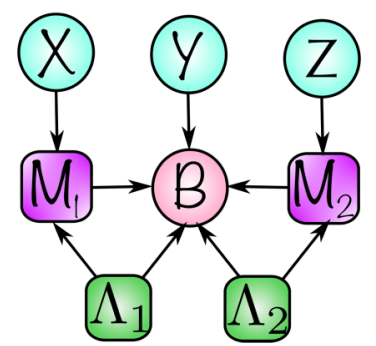

FIG. 6. (a) A black-box representation of a communication network with two preparation devices and a central measurement device (possibly measuring the incoming physical systems in a joint basis). (b) The DAG representation of the associated (classical) causal structure. Variables $M_{x}$ and $M_{z}$ stand for the prepared states that in the quantum case would be represented by preparing states $\rho_{x}$ and $\rho_{z}$. The variable $\Lambda$ represents the possible shared correlations between the devices and in a quantum description would be represented by a shared (possibly) entangled quantum state. (c) The DAG representation of the associated (classical) causal structure where there are two independent sources of correlations between the measurement and preparation devices.

many copies of it are distributed in a star-shaped network. As can be seen in Fig. 5(b), the entanglement swapping network can be seen as the combination of two standard bipartite Bell networks. In other terms, the Bell network can be seen as a building block to construct networks of growing size and complexity, on which the nonclassical nature of the states can be activated. In the following we will point out that the PAM causal structure can also be seen as a fundamental building block and investigate whether it can lead to activation results similar to the ones described above for Bell nonlocality.

\section{A. Two preparation devices and one measurement device}

We start by considering a communication quantum network, depicted in Fig. 6 and composed of two preparation devices and one measurement device. Assuming the devices might share correlations [see Fig. 6(b)], a classical description of it implies that the observed distribution $p(b \mid x, y, z)$ of such experiment can be decomposed as

$$
\begin{aligned}
p(b \mid x, y, z)= & \sum_{\lambda, m_{1}, m_{2}} p\left(m_{1} \mid x, \lambda\right) p\left(m_{2} \mid z, \lambda\right) \\
& \times p\left(b \mid m_{1}, m_{2}, y, \lambda\right) p(\lambda) .
\end{aligned}
$$

Clearly, if one of the preparation devices is traced out, we recover the standard prepare-and-measure scenario (Fig. 1), thus described by marginal distributions $p(b \mid x, y)$ or $p(b \mid y, z)$. Ideally, we would like to show that such scenario allows for activation of quantum resources, that is, resources that individually can only generate classical correlations [as described by the criterion (13) or (31)] but when distributed into a network become incompatible with the classical description (60). From the probability distribution perspective, this means that $p(b \mid x, y)$ and $p(b \mid y, z)$ have a classical decomposition but not $p(b \mid x, y, z)$.

To find activation, we have to employ quantum resources not violating the criterion (13) or (31) for the standard prepare-and-measure scenario but that do violate inequalities implied by the classical decomposition (60).

In the case where the messages are quantum, Born's prescription implies that

$$
p(b \mid x, y, z)=\operatorname{Tr}\left[\left(\rho_{x} \otimes \rho_{z}\right) M_{b}^{y}\right],
$$

where $M_{b}^{y}$ is now a measurement operator, potentially in an entangled basis, acting on both incoming qubits. In this case, we are interested in a situation where the prepared states $\rho_{x}$ and $\rho_{z}$ are classical [that is, do not violate (13)] but can have their nonclassical behavior witnessed when distributed in this network.

Considering the case where the exchanged messages are classical but the preparation and measurement devices share quantum correlations, we would like to have two independent states $\rho_{A B}$ and $\rho_{B C}$ that are classical in the standard PAM scenario [that is, do not violate (31)] but become nonclassical when distributed in the PAM network. The quantum description is then given by

$p(b \mid x, y, z)=\sum_{a, c} \operatorname{Tr}\left[\left(M_{a}^{x} \otimes M_{b}^{y, m(a), m(c)} \otimes M_{c}^{z}\right)\left(\rho_{A B} \otimes \rho_{B C}\right)\right]$.

To that aim, we first have to derive new inequalities characterizing the classical correlations in such a network as described by Eq. (60). In the simplest case where there are only two preparations $(|x|=|z|=2)$ and one single measurement $(|x|=1)$, a complete characterization of the classical inequalities have been obtained in [28]. We have also considered scenarios with a higher number of preparations $(|x|=$ $|z|=3)$ and measurements $(|y|=2)$. We have resorted to standard convex optimization algorithms to characterize such inequalities (see Appendix). However, in spite of its apparent simplicity, we were not able to achieve a full characterization of the classical correlations (60). Notwithstanding, we obtained a partial list of facets of the polytope described by (60); one of such inequalities being given by

$$
\sum_{x, y, z} \gamma_{x y z} E_{x y z} \leqslant 27
$$

where $E_{x y z}:=P(0 \mid x, y, z)-P(1 \mid x, y, z)$, and the coefficients $\gamma_{x y z}$ are

\begin{tabular}{c|ccc}
$\gamma_{x 1 z}$ & $z=1$ & $z=2$ & $z=3$ \\
\hline$x=1$ & -6 & -3 & 0 \\
$x=2$ & -2 & 2 & -3 \\
$x=3$ & 0 & -3 & 6 \\
$\gamma_{x 2 z}$ & $z=1$ & $z=2$ & $z=3$ \\
\hline$x=1$ & 3 & -3 & 1 \\
$x=2$ & -3 & 0 & -1 \\
$x=3$ & 3 & -3 & -3
\end{tabular}.

Unfortunately, by considering both the scenarios (61) and (62) and all the inequalities described above, we were 
unable to find any activation results (see Appendix for details on one numerical method). A possible origin of this negative result might be the fact that while in the quantum realization (61) and (62), the correlations between the preparation and measurement devices are limited, the classical description (60) allows for more general correlations. In this sense we are giving the classical description more explanation power.

For instance, in (61) the preparation and measurement devices are completely uncorrelated. For a fair comparison one should forbid such correlations in the classical model that would be then described by

$$
\begin{aligned}
& p(b \mid x, y, z)=\sum_{\mu_{1}, \mu_{2}, v, m_{1}, m_{2}} p\left(m_{1} \mid x, \mu_{1}\right) p\left(m_{2} \mid z, \mu_{2}\right) \\
& p\left(b \mid m_{1}, m_{2}, y, v\right) p\left(\mu_{1}\right) p\left(\mu_{2}\right) p(\nu) .
\end{aligned}
$$

Notice that the independence of the sources implies a nonlinear constraint $p\left(\mu_{1}, \mu_{2}, v\right)=p\left(\mu_{1}\right) p\left(\mu_{2}\right) p(v)$ and thus the set of classically allowed correlations is not a polytope anymore, making it difficult to attack the problem [8,27].

In turn, in (62) we are using two independent sources of quantum states. However, the classical description (60) allows for a single source of correlations. As discussed before, a more fair comparison would be to consider the classical model given by [see Fig. 6(c)]

$$
\begin{aligned}
& p(b \mid x, y, z)=\sum_{\lambda_{1}, \lambda_{2}, m_{1}, m_{2}} p\left(m_{1} \mid x, \lambda_{1}\right) p\left(m_{2} \mid z, \lambda_{2}\right) \\
& p\left(b \mid m_{1}, m_{2}, y, \lambda_{1}, \lambda_{2}\right) p\left(\lambda_{1}\right) p\left(\lambda_{2}\right) .
\end{aligned}
$$

Notice that this model is similar to the bilocality scenario within the context of Bell's nonlocality [6]. As in the bilocality case, the nonlinear constraint $p\left(\lambda_{1}, \lambda_{2}\right)=p\left(\lambda_{1}\right) p\left(\lambda_{2}\right)$ implies that the set of classicality-allowed correlations is nonconvex, once again making it very difficult to be characterized.

\section{B. Combining different building blocks}

One can also combine different kinds of building blocks. With a building block we mean a causal structure/network that by itself can lead to a gap in the classical versus quantum description of correlations. To our knowledge there are so far three of these: the Bell [3], the PAM [16], and the instrumental [51] causal structures.

For instance, as shown in Fig. 7, one can combine the Bell and PAM causal structures. Assuming that all the devices share correlations the classical description is given by

$$
p(a, b \mid x, y, z)=\sum_{\lambda, m} p(a \mid x, \lambda) p(b \mid y, m, \lambda) p(m \mid z, \lambda) p(\lambda) .
$$

Focusing on full correlators $\left\langle A_{x} B_{y z}\right\rangle=p(a=b \mid x, y, z)-$ $p(a \neq b \mid x, y, z)$ (with $a, b \in\{0,1\})$ we have obtained a full characterization of the associated polytope for $|x|=|y|=2$ and $|z|=3$. As described in the Appendix, there are a total of 17 nontrivial Bell inequalities. Looking for activation of quantum resources we have focused on the following scenario: two quantum states $\rho_{A B}$ and $\rho_{B C}$, such that $\rho_{A B}$ does not violate the Horodecki criterion [36] and $\rho_{B C}$ does not violate the criterion (31), but that potentially can violate one (a)

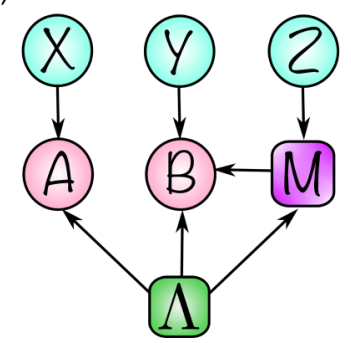

(b)

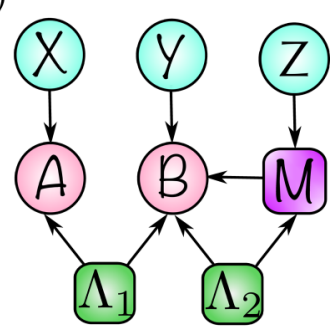

FIG. 7. (a) Causal structure using as building blocks a Bell and a PAM scenario. In this case, it is assumed that all devices might share correlations described by the variable $\Lambda$. (b) The Bell-PAM scenario, but in this case assuming that the correlations are mediated by two independent sources.

of these 17 nonequivalent classes of inequalities for the combined scenario. Unfortunately, by optimizing over states and measurements we could not find any examples.

\section{DISCUSSION}

Arguably, one of nearest-term offsprings of quantum technologies is quantum communication [52], the relevance of which stems from the fact that quantum resources improve our capabilities to communicate more efficiently and more securely. Recent milestones, such as the first loophole-free violation of Bell inequalities [53-55] and the launch of a satellite establishing quantum channels across intercontinental distances [56], are now paving the way to the establishment of the networks of growing size and complexity. Understanding the advantages provided by quantum resources is thus an important and necessary step.

In this work we have considered the paradigmatic prepareand-measure scenario [16] and possible extensions of it into a network. By considering the simplest nontrivial PAM scenario we have derived a set of criteria testing whether a given quantum resource is compatible or not with a classical description. More precisely, we derived conditions for the quantum violation of the dimension witness inequality introduced in [16], considering that both the prepared states and the shared correlations between the devices are quantum. These criteria are the analog for the prepare-and-measure scenario of what the Hororecki criterion [36] is for the Bell scenario.

Based on that, we have proven a number of activation-like results showing how quantum resources having a classical description in the simplest PAM scenario become nonclassical if the number of either preparations or measurements is increased. Further, we have discussed how the PAM scenario can be considered as a building block for more complex communication networks. In the case of a network, we were unable to find any activation results. However, as mentioned, this is possibly due to the fact that in order to be able to derive inequalities to be violated, we are allowing the classical models to have shared correlations between all devices. Understanding how to derive nonlinear inequalities [27] for communication scenarios is certainly interesting by its own and could lead to activation in communication networks. 
Finally, it is worth mentioning that here we have focused on the simplest possible PAM scenario. As in the Bell case [38,39], it would be relevant to come up with general methods witnessing that a given set of quantum states can only give rise to classical correlations, irrespective of the number of measurement being performed. We hope that our result might trigger future research in this direction.

\section{ACKNOWLEDGMENTS}

We acknowledge the John Templeton Foundation via the Grant Q-CAUSAL No. 61084, the Serrapilheira Institute (Grant No. Serra-1708-15763), the Brazilian National Council for Scientific and Technological Development (CNPq) via the National Institute for Science and Technology on Quantum Information (INCT-IQ) and Grants No. 307172/2017-1 and No. 406574/2018-9, and the Brazilian agencies MCTIC and MEC. D.P. acknowledges Sapienza Università di Roma via the grant for joint research projects for mobility No. 2289/2018 Prot. No. 50074.

\section{APPENDIX}

\section{Derivation of dimension witness inequalities [PAM scenario} $(|x|,|y|)$ for $(3,3)$ and $(4,2)]$

To derive the witness inequalities for the prepare-andmeasure scenario shown in Fig. 1(b) (see also Fig. 4), first we listed all the extremal points (V representation) of the polytope associated with the causal structure in Fig. 1(b). We allow the preparation and measurement devices to share correlations, so any distribution compatible with that causal structure has a decomposition given by

$$
p(b \mid x, y)=\sum_{m, \lambda} p(b \mid m, y, \lambda) p(m \mid x, \lambda) p(\lambda) .
$$

The terms in the summation are deterministic functions that can be written as $p(b \mid m, y, \lambda)=\delta_{b, f(m, y, \lambda)}$ and $p(m \mid x, \lambda)=\delta_{m, g(x, \lambda)}$, where $f($.$) and g($.$) are Boolean func-$ tions with $|m|=2$ (that is, the prepared states are binary), $|x|=3(4)$, and $|y|=3(2)$. We found 512 extremal points for the $(3,3)$ scenario and 256 for $(4,2)$, where (.,.) stands for $(|x|,|y|)$.

To derive the inequalities we used the "lrs.c" [57] program which provides us the facets of the polytope and has as input the $\mathrm{V}$ representation of it. The inequalities were determined using the set of the variables given by the correlators $\left\{B_{x, y}\right\}$ which give us a total of $|x| \times|y|$ variables. We could completely characterize these two scenarios finding 186 inequalities for $(3,3)$ and 136 for the $(4,2)$ polytopes. Considering the symmetries of each inequality, given by permutation of inputs or outputs, we found 2 nontrivial inequality classes for the $(4,2)$ scenario and 3 classes for the $(3,3)$ scenario. These classes are shown in Tables I and II.

\section{Derivation of dimension witness inequalities (PAM-PAM scenario)}

To derive the witness inequalities for the combined prepare-and-measure scenario shown in Fig. 6(a), we need to find all the extremal points ( $\mathrm{V}$ representation) of the poly-
TABLE I. Inequality classes obtained for PAM $(3,3)$.

\begin{tabular}{lc}
\hline \hline Class & Inequality PAM Scenario $(3,3)$ \\
\hline 1 & $E_{11}+E_{12}+E_{21}-E_{22}-E_{31} \leqslant 3$ \\
2 & $E_{11}+E_{12}-E_{22}+E_{23}-E_{31}-E_{33} \leqslant 4$ \\
3 & $E_{11}+E_{12}+E_{13}+E_{21}-E_{22}-E_{23}-E_{31}+E_{32}-E_{33} \leqslant 5$ \\
\hline \hline
\end{tabular}

tope associated with the causal structure in Fig. 6(b). The preparation and measurement devices are allowed to share correlations; then any distribution compatible with that causal structure has a decomposition given by

$$
\begin{array}{r}
p(b \mid x, y, z)=\sum_{m_{1}, m_{2}, \lambda} p\left(b \mid m_{1}, m_{2}, y, \lambda\right) \\
p\left(m_{1} \mid x, \lambda\right) p\left(m_{2} \mid z, \lambda\right) p(\lambda) .
\end{array}
$$

The terms in the summation are deterministic functions that can be written as $p\left(b \mid m_{1}, m_{2}, y, \lambda\right)=\delta_{b, f\left(m_{1}, m_{2}, y, \lambda\right)}$, $p\left(m_{1} \mid x, \lambda\right)=\delta_{m_{1}, g_{1}(x, \lambda)}$, and $p\left(m_{2} \mid z, \lambda\right)=\delta_{m_{2}, g_{2}(z, \lambda)}$, where $f(),. g_{1}($.$) , and g_{2}($.$) are Boolean functions. The polytope is$ characterized by 2128 extremal points.

To derive the inequalities we used the "Irs.c" [57] program which provides us the facets of the polytope and has as input the $\mathrm{V}$ representation of it. We are considering the case of three preparations $(|x|=|z|=3)$ and two measurements $(|y|=2)$ We could not fully characterize the polytope, but we could find 8619 facets for this scenario. One example of these inequalities is provided in the main text. Notice that in [28] a complete characterization has been found; however, in that paper there is a single possible measurement $(|y|=1)$.

\section{Derivation of dimension witness inequalities (Bell-PAM scenario)}

The classical description of the combined Bell and PAM scenario (see Fig. 7) is given by

$$
\begin{aligned}
p(a, b \mid x, y, z)= & \sum_{m \lambda} p(a \mid x, \lambda) p(b \mid m, y, \lambda) \\
& p(m \mid z, \lambda) p(\lambda) .
\end{aligned}
$$

To derive the corresponding inequalities bounding this scenario we need to find all the extremal points (V representation) of the polytope associated with this causal structure.

The terms in the summation are deterministic functions that can be written as $p(a \mid x, \lambda)=\delta_{a, f_{1}(a, x, \lambda)}, p(b \mid m, y, \lambda)=$ $\delta_{b, f_{2}(m, y, \lambda)}$, and $p(m \mid z, \lambda)=\delta_{m, g(z, \lambda)}$, where $f_{1}(),. f_{2}($.$) , and$ $g($.$) are Boolean functions. We are considering the case |x|=$ $|y|=|a|=|b|=|m|=2$ and $|z|=3$ only for full correlators. We have a set of 12 variables given by $\left\{E_{x y z}\right\}$ and this polytope is characterized by 80 extremal points.

TABLE II. Inequality classes obtained for PAM $(4,2)$.

\begin{tabular}{lc}
\hline \hline Class & Inequality PAM Scenario (4,2) \\
\hline 1 & $E_{11}+E_{12}+E_{21}-E_{22}-E_{41} \leqslant 3$ \\
2 & $E_{11}+E_{12}+E_{21}-E_{22}-E_{31}+E_{32}-E_{41}-E_{42} \leqslant 4$ \\
\hline \hline
\end{tabular}


TABLE III. Inequality classes obtained for Bell-PAM $(2,2,3)$ for full correlators.

\begin{tabular}{lc}
\hline \hline Class & Inequality Bell-PAM Scenario $(2,2,3)$ \\
\hline 1 & $E_{111}+E_{112}+E_{211}-E_{212} \leqslant 2$ \\
2 & $E_{111}+E_{112}-E_{113}+E_{121}-E_{122} \leqslant 3$ \\
3 & $4 E_{111}+E_{112}-E_{113}+E_{121}-E_{122}+2 E_{211}$ \\
& $-E_{212}+E_{213}-E_{221}+E_{222} \leqslant 6$ \\
4 & $3 E_{111}+E_{112}-E_{113}+E_{121}-E_{122}-E_{123}+3 E_{211}$ \\
& $-E_{212}+E_{213}-E_{221}+E_{222}+E_{223} \leqslant 6$ \\
5 & $2 E_{111}+E_{112}-2 E_{113}+2 E_{121}$ \\
& $-2 E_{122}+E_{123}+E_{212}-E_{223} \leqslant 6$ \\
\hline \hline
\end{tabular}

To derive the inequalities we used the "lrs.c" [57] program which provides us the facets of the polytope and has as input the $\mathrm{V}$ representation of it. We could fully characterize the polytope; we find 1296 facets for this scenario divided into 5 nontrivial classes shown in Table III.

\section{Alternative derivation of criterion (22)}

Take inequality (20) as our starting point, restated here for convenience as

$$
\begin{aligned}
S= & \frac{1}{2}\left[\left(\boldsymbol{q}_{1}^{A}+\boldsymbol{q}_{2}^{A}-\boldsymbol{q}_{3}^{A}\right) T\left(\boldsymbol{q}_{10}^{B}-\boldsymbol{q}_{11}^{B}\right)\right. \\
& \left.+\left(\boldsymbol{q}_{1}^{A}-\boldsymbol{q}_{2}^{A}\right) T\left(\boldsymbol{q}_{20}^{B}-\boldsymbol{q}_{21}^{B}\right)+\left(\boldsymbol{q}_{11}^{B}+\boldsymbol{q}_{10}^{B}\right) \cdot \boldsymbol{s}\right],
\end{aligned}
$$

using directly $b=0,1$ instead of $m=(-1)^{b}$. Assume now $\boldsymbol{s}=0$ and that all measurements are projective. We should optimize $S$ with the restrictions that every vector in the expression has unit norm. The Lagrangian for this problem is given by

$$
\Lambda:=S+\sum_{i=1}^{3} \lambda_{i}^{A}\left(\boldsymbol{q}_{i}^{A} \cdot \boldsymbol{q}_{i}^{A}-1\right)+\sum_{i=1}^{2} \sum_{m=0}^{1} \lambda_{i m}^{B}\left(\boldsymbol{q}_{i m}^{B} \cdot \boldsymbol{q}_{i m}^{B}-1\right) .
$$

Our goal is then to find a solution for $\nabla \Lambda=0$. Solving for $\boldsymbol{q}_{3}^{A}$ first, we get the condition

$$
-T\left(\boldsymbol{q}_{10}^{B}-\boldsymbol{q}_{11}^{B}\right)+2 \lambda_{3}^{A} \boldsymbol{q}_{3}^{A}=0,
$$

which implies $\boldsymbol{q}_{3}^{A}$ parallel to $T\left(\boldsymbol{q}_{10}^{B}-\boldsymbol{q}_{11}^{B}\right)$ and, thus, that the original inequality should become

$$
\begin{aligned}
S= & \frac{1}{2}\left[\left(\boldsymbol{q}_{1}^{A}+\boldsymbol{q}_{2}^{A}\right) T\left(\boldsymbol{q}_{10}^{B}-\boldsymbol{q}_{11}^{B}\right)+\left\|T\left(\boldsymbol{q}_{10}^{B}-\boldsymbol{q}_{11}^{B}\right)\right\|\right. \\
& \left.+\left(\boldsymbol{q}_{1}^{A}-\boldsymbol{q}_{2}^{A}\right) T\left(\boldsymbol{q}_{20}^{B}-\boldsymbol{q}_{21}^{B}\right)\right],
\end{aligned}
$$

where we have also considered the assumption of $s=0$. We may now make $2 \boldsymbol{u}_{1} \cos (\theta)=\boldsymbol{q}_{1}^{A}+\boldsymbol{q}_{2}^{A}$ and $2 \boldsymbol{u}_{2} \sin (\theta)=\boldsymbol{q}_{1}^{A}-$ $\boldsymbol{q}_{2}^{A}$, with $\left\|u_{i}\right\|=1, \quad(i=1,2)$, and $\boldsymbol{u}_{1}^{T} \cdot \boldsymbol{u}_{2}=0$. We may also make $2 \boldsymbol{v} \cos (\alpha)=\boldsymbol{q}_{10}^{B}-\boldsymbol{q}_{11}^{B}$ and $2 \boldsymbol{w} \cos (\beta)=\boldsymbol{q}_{20}^{B}-\boldsymbol{q}_{21}^{B}$, with $\|\boldsymbol{v}\|=\|\boldsymbol{w}\|=1$. We then get

$$
\begin{aligned}
S= & 2\left[\boldsymbol{u}_{1} T \boldsymbol{v} \cos (\theta) \cos (\alpha)+\frac{1}{2}\|T \boldsymbol{v}\||\cos (\alpha)|\right. \\
& \left.+\boldsymbol{u}_{2} T \boldsymbol{w} \sin (\theta) \cos (\beta)\right] ;
\end{aligned}
$$

we may choose $\alpha=\beta=0$ and make each term positive with the other variables. By optimizing over $\theta$, we get

$$
S=2\left[\sqrt{\left(\boldsymbol{u}_{1} T \boldsymbol{v}\right)^{2}+\left(\boldsymbol{u}_{2} T \boldsymbol{w}\right)^{2}}+\frac{1}{2}\|T \boldsymbol{v}\|\right] .
$$

We may consider $T$ diagonal, by embedding into $\boldsymbol{v}, \boldsymbol{w}$, and $\boldsymbol{u}_{i}$ the necessary rotations to realize its singular value decomposition. In particular, let $T=\sum_{i=1}^{3} \mu_{i} \varepsilon_{i} \otimes \varepsilon_{i}^{T}$, where $\left|\mu_{1}\right| \geqslant\left|\mu_{2}\right| \geqslant\left|\mu_{3}\right|$, and $\varepsilon_{i}$ the eigenvector associated to the eigenvalue $\mu_{i}$. The maximal value for $S$ is then obtained by setting $\boldsymbol{v}=\boldsymbol{u}_{1}=\varepsilon_{1}$ and $\boldsymbol{w}=\boldsymbol{u}_{2}=\varepsilon_{2}$, in which case, we obtain

$$
S=2 \sqrt{\mu_{1}^{2}+\mu_{2}^{2}}+\left|\mu_{1}\right|,
$$

which coincides with the criterion established in (22), noting that $\mu_{i}^{2}=\lambda_{i}$. In particular, note that $T \boldsymbol{v}=\mu_{1} \varepsilon_{1}$, which implies $\boldsymbol{q}_{3}^{A}=-\frac{\mu_{1}}{\left|\mu_{i}\right|} \varepsilon_{1}$. Also, since $\boldsymbol{q}_{1}^{A}+\boldsymbol{q}_{2}^{A}=2 \cos (\theta) \varepsilon_{1}$, we retrieve our original assumption that $\boldsymbol{q}_{3}^{A}$ should be parallel to $\boldsymbol{q}_{1}^{A}+\boldsymbol{q}_{2}^{A}$.

\section{Optimization details to find nonclassicality activation}

In this section, we describe the method used to look for nonclassicality activation in the combined scenario of two preparation devices and one measurement device [illustrated in Fig. 6(b)]. Restating the problem here, we are interested in finding sets of density matrices $\left\{\rho_{x}^{A}\right\}_{x}$ and $\left\{\rho_{z}^{C}\right\}_{z}$ that, in the simple prepare-and-measure scenario, cannot produce nonclassical probabilities regardless of the measurements performed in the receiving apparatus, but that, when combined, present a nonclassical correlation in the bigger setup.

Recall that each set of density matrices must satisfy the following criteria to produce only classical distributions:

$$
\begin{aligned}
& \left\|\boldsymbol{q}_{1}^{P}+\boldsymbol{q}_{2}^{P}-\boldsymbol{q}_{3}^{P}\right\|+\left\|\boldsymbol{q}_{1}^{P}-\boldsymbol{q}_{2}^{P}\right\| \leqslant 3, \\
& \left\|\boldsymbol{q}_{1}^{P}+\boldsymbol{q}_{3}^{P}-\boldsymbol{q}_{2}^{P}\right\|+\left\|\boldsymbol{q}_{1}^{P}-\boldsymbol{q}_{3}^{P}\right\| \leqslant 3, \\
& \left\|\boldsymbol{q}_{2}^{P}+\boldsymbol{q}_{3}^{P}-\boldsymbol{q}_{1}^{P}\right\|+\left\|\boldsymbol{q}_{2}^{P}-\boldsymbol{q}_{3}^{P}\right\| \leqslant 3,
\end{aligned}
$$

where $\boldsymbol{q}_{j}^{P}=\operatorname{Tr}\left[\rho_{j}^{P} \boldsymbol{\sigma}\right] ; P=A, C ; j=1,2,3$. Since each inequality is symmetric under a rigid rotation of all vectors $\boldsymbol{q}_{i}^{P}$ of a given party, we may fixate $\boldsymbol{q}_{1}^{P}=\boldsymbol{z}$ and take $\boldsymbol{q}_{2}^{P}$ on the $x z$ plane. Assuming pure states only, this leaves us with three parameters per party to determine a set of states and viable candidates for the activation task (note that the simplified form of the criteria [Eq. (15)] requires also three parameters, however with a different interpretation than the ones used here).

We establish a region of viable candidates for activation. Using $\boldsymbol{q}_{2}^{P}=\cos (\varphi) \boldsymbol{x}+\sin (\varphi) \boldsymbol{z}$ and $\boldsymbol{q}_{3}^{P}=\sin (\theta) \cos (\phi) \boldsymbol{x}+$ $\sin (\theta) \sin (\phi) \boldsymbol{y}+\cos (\theta) z$, we filter the candidates by generating a grid of triplets $(\varphi, \phi, \theta)$ that correspond to vectors that satisfy all criteria of Eq. (A11). We then sample candidates within the grid, and optimize Bob's measurement over each candidate via a semidefinite program:

$$
\begin{array}{ll}
\text { Given } & \beta_{b x y z}, \rho_{x}^{A}, \rho_{z}^{C}, \\
\underset{M_{b}^{y}}{\operatorname{maximize}} & \sum_{b, x, y, z} \beta_{b x y z} \operatorname{Tr}\left[M_{b}^{y} \rho_{x}^{A} \otimes \rho_{z}^{C}\right], \\
\text { subject to } & M_{b}^{y} \geqslant 0, \\
& \sum_{b=0}^{1} M_{b}^{y}=\mathbb{1},
\end{array}
$$


where $\beta_{b x y z}$ are the coefficients of a Bell-like inequality for the combined scenario of two preparing devices and one measur- ing device. $\rho_{x}^{A}$ and $\rho_{z}^{C}$ are obtained from the sampled Bloch vectors.
[1] J. Pearl, Causality (Cambridge University Press, Cambridge, England, 2009).

[2] P. Spirtes, C. N. Glymour, R. Scheines, D. Heckerman, C. Meek, G. Cooper, and T. Richardson, Causation, Prediction, and Search (MIT Press, Cambridge, Massachusetts, 2000).

[3] J. S. Bell, On the Einstein-Podolsky-Rosen paradox, Physics 1, 195 (1964).

[4] C. J. Wood and R. W. Spekkens, The lesson of causal discovery algorithms for quantum correlations: Causal explanations of Bell-inequality violations require fine-tuning, New J. Phys. 17, 033002 (2015).

[5] R. Chaves, R. Kueng, J. B. Brask, and D. Gross, Unifying Framework for Relaxations of the Causal Assumptions in Bell's Theorem, Phys. Rev. Lett. 114, 140403 (2015).

[6] C. Branciard, N. Gisin, and S. Pironio, Characterizing the Nonlocal Correlations Created via Entanglement Swapping, Phys. Rev. Lett. 104, 170401 (2010).

[7] T. Fritz, Beyond Bell's theorem: Correlation scenarios, New J. Phys. 14, 103001 (2012).

[8] R. Chaves, Polynomial Bell Inequalities, Phys. Rev. Lett. 116, 010402 (2016).

[9] M.-O. Renou, E. Bäumer, S. Boreiri, N. Brunner, N. Gisin, and S. Beigi, Genuine Quantum Nonlocality in the Triangle Network, Phys. Rev. Lett. 123, 140401 (2019).

[10] G. Carvacho, F. Andreoli, L. Santodonato, M. Bentivegna, R. Chaves, and F. Sciarrino, Experimental violation of local causality in a quantum network, Nat. Commun. 8, 14775 (2017).

[11] D. J. Saunders, A. J. Bennet, C. Branciard, and G. J. Pryde, Experimental demonstration of nonbilocal quantum correlations, Sci. Adv. 3, e1602743 (2017).

[12] Q.-C. Sun, Y.-F. Jiang, B. Bai, W. Zhang, H. Li, X. Jiang, J. Zhang, L. You, X. Chen, Z. Wang et al., Experimental demonstration of non-bilocality with truly independent sources and strict locality constraints, Nat. Photonics 13, 687 (2019).

[13] G. Carvacho, R. Chaves, and F. Sciarrino, Perspective on experimental quantum causality, Europhys. Lett. 125, 30001 (2019).

[14] C. H. Bennett, G. Brassard, C. Crépeau, R. Jozsa, A. Peres, and W. K. Wootters, Teleporting an Unknown Quantum State via Dual Classical and Einstein-Podolsky-Rosen Channels, Phys. Rev. Lett. 70, 1895 (1993).

[15] C. H. Bennett and S. J. Wiesner, Communication via Oneand Two-Particle Operators on Einstein-Podolsky-Rosen States, Phys. Rev. Lett. 69, 2881 (1992).

[16] R. Gallego, N. Brunner, C. Hadley, and A. Acín, DeviceIndependent Tests of Classical and Quantum Dimensions, Phys. Rev. Lett. 105, 230501 (2010).

[17] A. Ambainis, A. Nayak, A. Ta-Shma, and U. Vazirani, Dense quantum coding and quantum finite automata, J. Assoc. Comput. Mach. 49, 496 (2002).

[18] M. Pawłowski and M. Żukowski, Entanglement-assisted random access codes, Phys. Rev. A 81, 042326 (2010).

[19] A. Hameedi, D. Saha, P. Mironowicz, M. Pawłowski, and M. Bourennane, Complementarity between entanglement-assisted and quantum distributed random access code, Phys. Rev. A 95 052345 (2017).

[20] M. Pawłowski, T. Paterek, D. Kaszlikowski, V. Scarani, A. Winter, and M. Żukowski, Information causality as a physical principle, Nature (London) 461, 1101 (2009).

[21] R. Chaves, C. Majenz, and D. Gross, Information-theoretic implications of quantum causal structures, Nat. Commun. 6, 5766 (2015).

[22] R. Chaves, G. B. Lemos, and J. Pienaar, Causal Modeling the Delayed-Choice Experiment, Phys. Rev. Lett. 120, 190401 (2018).

[23] E. Polino, I. Agresti, D. Poderini, G. Carvacho, G. Milani, G. B. Lemos, R. Chaves, and F. Sciarrino, Device-independent test of a delayed choice experiment, Phys. Rev. A 100, 022111 (2019).

[24] S. Yu, Y.-N. Sun, W. Liu, Z.-D. Liu, Z.-J. Ke, Y.-T. Wang, J.-S. Tang, C.-F. Li, and G.-C. Guo, Realization of a causal-modeled delayed-choice experiment using single photons, Phys. Rev. A 100, 012115 (2019).

[25] H.-L. Huang, Y.-H. Luo, B. Bai, Y.-H. Deng, H. Wang, Q. Zhao, H.-S. Zhong, Y.-Q. Nie, W.-H. Jiang, X.-L. Wang, J. Zhang, L. Li, N.-L. Liu, T. Byrnes, J. P. Dowling, C.-Y. Lu, and J.-W. Pan, Compatibility of causal hidden-variable theories with a delayed-choice experiment, Phys. Rev. A 100, 012114 (2019).

[26] R. Chaves, J. B. Brask, and N. Brunner, Device-Independent Tests of Entropy, Phys. Rev. Lett. 115, 110501 (2015).

[27] J. Bowles, M. T. Quintino, and N. Brunner, Certifying the Dimension of Classical and Quantum Systems in a Prepare-andMeasure Scenario with Independent Devices, Phys. Rev. Lett. 112, 140407 (2014).

[28] J. Bowles, N. Brunner, and M. Pawłowski, Testing dimension and nonclassicality in communication networks, Phys. Rev. A 92, 022351 (2015).

[29] J. I. de Vicente, A general bound for the dimension of quantum behaviours in the prepare-and-measure scenario, J. Phys. A Math. Theor. 52, 095304 (2019).

[30] Y. Wang, I. W. Primaatmaja, E. Lavie, A. Varvitsiotis, and Charles $\mathrm{Ci}$ Wen Lim, Characterising the correlations of prepareand-measure quantum networks, npj Quantum Inf. 5, 17 (2019).

[31] M. Pawłowski and N. Brunner, Semi-device-independent security of one-way quantum key distribution, Phys. Rev. A 84, 010302(R) (2011).

[32] A. Tavakoli, J. Kaniewski, T. Vértesi, D. Rosset, and N. Brunner, Self-testing quantum states and measurements in the prepare-and-measure scenario, Phys. Rev. A 98, 062307 (2018).

[33] E. Woodhead and S. Pironio, Secrecy in Prepare-and-Measure Clauser-Horne-Shimony-Holt Tests with a Qubit Bound, Phys. Rev. Lett. 115, 150501 (2015).

[34] T. Lunghi, J. B. Brask, C. C. W. Lim, Q. Lavigne, J. Bowles, A. Martin, H. Zbinden, and N. Brunner, Self-Testing Quantum Random Number Generator, Phys. Rev. Lett. 114, 150501 (2015).

[35] W. Shi, Yu Cai, J. B. Brask, H. Zbinden, and N. Brunner, Semidevice-independent characterization of quantum measurements 
under a minimum overlap assumption, Phys. Rev. A 100, 042108 (2019).

[36] R. Horodecki, P. Horodecki, and M. Horodecki, Violating Bell inequality by mixed spin- $\frac{1}{2}$ states: Necessary and sufficient condition, Phys. Lett. A 200, 340 (1995).

[37] J. F. Clauser, M. A. Horne, A. Shimony, and R. A. Holt, Proposed Experiment to Test Local Hidden-Variable Theories, Phys. Rev. Lett. 23, 880 (1969).

[38] D. Cavalcanti, L. Guerini, R. Rabelo, and P. Skrzypczyk, General Method for Constructing Local Hidden Variable Models for Entangled Quantum States, Phys. Rev. Lett. 117, 190401 (2016).

[39] F. Hirsch, M. T. Quintino, T. Vértesi, M. F. Pusey, and N. Brunner, Algorithmic Construction of Local Hidden Variable Models for Entangled Quantum States, Phys. Rev. Lett. 117, 190402 (2016).

[40] F. Andreoli, G. Carvacho, L. Santodonato, R. Chaves, and F. Sciarrino, Maximal qubit violation of n-locality inequalities in a star-shaped quantum network, New J. Phys. 19, 113020 (2017).

[41] N. Gisin, Q. Mei, A. Tavakoli, M. O. Renou, and N. Brunner, All entangled pure quantum states violate the bilocality inequality, Phys. Rev. A 96, 020304(R) (2017).

[42] D. Cavalcanti, P. Skrzypczyk, and I. Šupić, All Entangled States Can Demonstrate Nonclassical Teleportation, Phys. Rev. Lett. 119, 110501 (2017).

[43] A. Tavakoli, E. Z. Cruzeiro, J. B. Brask, N. Gisin, and N. Brunner, Informationally restricted quantum correlations, Quantum 4, 332 (2020).

[44] R. Horodecki and M. Horodecki, Information-theoretic aspects of inseparability of mixed states, Phys. Rev. A 54, 1838 (1996).

[45] D. Collins and N. Gisin, A relevant two qubit Bell inequality inequivalent to the CHSH inequality, J. Phys. A: Math. Gen. 37, 1775 (2004)

[46] M. T. Quintino, T. Vértesi, and N. Brunner, Joint Measurability, Einstein-Podolsky-Rosen Steering, and Bell Nonlocality, Phys. Rev. Lett. 113, 160402 (2014).

[47] A. Wójcik, J. Modławska, A. Grudka, and M. Czechlewski, Violation of Clauser-Horne-Shimony-Holt inequality for states resulting from entanglement swapping, Phys. Lett. A 374, 4831 (2010).

[48] A. Sen(De), U. Sen, Č. Brukner, V. Bužek, and M. Żukowski, Entanglement swapping of noisy states: A kind of superadditivity in nonclassicality, Phys. Rev. A 72, 042310 (2005).
[49] W. Kłobus, W. Laskowski, M. Markiewicz, and A. Grudka, Nonlocality activation in entanglement-swapping chains, Phys. Rev. A 86, 020302(R) (2012).

[50] D. Cavalcanti, M. L. Almeida, V. Scarani, and A. Acin, Quantum networks reveal quantum nonlocality, Nat. Commun. 2, 184 (2011).

[51] R. Chaves, G. Carvacho, I. Agresti, V. Di Giulio, L. Aolita, S. Giacomini, and F. Sciarrino, Quantum violation of an instrumental test, Nat. Phys. 14, 291 (2018).

[52] N. Gisin and R. Thew, Quantum communication, Nat. Photonics 1, 165 (2007).

[53] B. Hensen, H. Bernien, A. E. Dréau, A. Reiserer, N. Kalb, M. S. Blok, J. Ruitenberg, R. F. L. Vermeulen, R. N. Schouten, C. Abellán et al., Loophole-free Bell inequality violation using electron spins separated by 1.3 kilometres, Nature (London) 526, 682 (2015).

[54] L. K. Shalm, E. Meyer-Scott, B. G. Christensen, P. Bierhorst, M. A. Wayne, M. J. Stevens, T. Gerrits, S. Glancy, D. R. Hamel, M. S. Allman, K. J. Coakley, S. D. Dyer, C. Hodge, A. E. Lita, V. B. Verma, C. Lambrocco, E. Tortorici, A. L. Migdall, Y. Zhang, D. R. Kumor, W. H. Farr, F. Marsili, M. D. Shaw, J. A. Stern, C. Abellán, W. Amaya, V. Pruneri, T. Jennewein, M. W. Mitchell, P. G. Kwiat, J. C. Bienfang, R. P. Mirin, E. Knill, and S. W. Nam, Strong Loophole-Free Test of Local Realism, Phys. Rev. Lett. 115, 250402 (2015).

[55] M. Giustina, M. A. M. Versteegh, S. Wengerowsky, J. Handsteiner, A. Hochrainer, K. Phelan, F. Steinlechner, J. Kofler, J. Larsson, C. Abellán, W. Amaya, V. Pruneri, M. W. Mitchell, J. Beyer, T. Gerrits, A. E. Lita, L. K. Shalm, S. W. Nam, T. Scheidl, R. Ursin, B. Wittmann, and A. Zeilinger, Significant-Loophole-Free Test of Bell's Theorem with Entangled Photons, Phys. Rev. Lett. 115, 250401 (2015).

[56] S.-K. Liao, W.-Q. Cai, J. Handsteiner, B. Liu, J. Yin, L. Zhang, D. Rauch, M. Fink, J.-G. Ren, W.-Y. Liu, Y. Li, Q. Shen, Y. Cao, F.-Z. Li, J.-F. Wang, Y.-M. Huang, L. Deng, T. Xi, L. Ma, T. Hu, L. Li, N.-L. Liu, F. Koidl, P. Wang, Y.-A. Chen, X.-B. Wang, M. Steindorfer, G. Kirchner, C.-Y. Lu, R. Shu, R. Ursin, T. Scheidl, C.-Z. Peng, J.-Y. Wang, A. Zeilinger, and J.-W. Pan, SatelliteRelayed Intercontinental Quantum Network, Phys. Rev. Lett. 120, 030501 (2018).

[57] D. Avis, K. Fukuda, and S. Picozzi, On canonical representations of convex polyhedra, in Mathematical Software (World Scientific, Singapore, 2002), pp. 350-360. 